\title{
Clinical practice alert on the use of immune checkpoint inhibitors in patients latently infected with tuberculosis
}

Chirag Dhar, Departments of Medicine and Cellular and Molecular Medicine, University of California, San Diego, La Jolla, USA. Email: cdhar@ucsd.edu

\begin{abstract}
Multiple case reports have been published on the risk of tuberculosis activation in patients on immune checkpoint inhibitors (ICPIS). To this matter, I caution clinicians to test for latent tuberculosis before starting their patients on ICPIs.
\end{abstract}

\section{Introduction}

It has been a year since the announcement of the Nobel prize in medicine and physiology for the discovery of immune checkpoint inhibitors (ICPIS) (1). ICPIs have indeed revolutionized cancer therapy as was suggested in the original award citation. But an important yet often overlooked phenomena when using ICPIs is the risk of infection reactivation due to immunosuppression. Activation of asymptomatic and undiagnosed latent tuberculosis (TB) is a significant concern as demonstrated by multiple case studies (2-8). One possible mechanism for reactivation of TB in patients on antiPD-1 therapy (9) is pictorially depicted in figure 1.

Figure 1: Pictorial depiction of one possible mechanism for TB activation by anti-PD-1 immunotherapy. Image created with objects sourced from Servier Medical Art (http://smart.servier.com/), licensed under a Creative Common Attribution 3.0 Generic License.

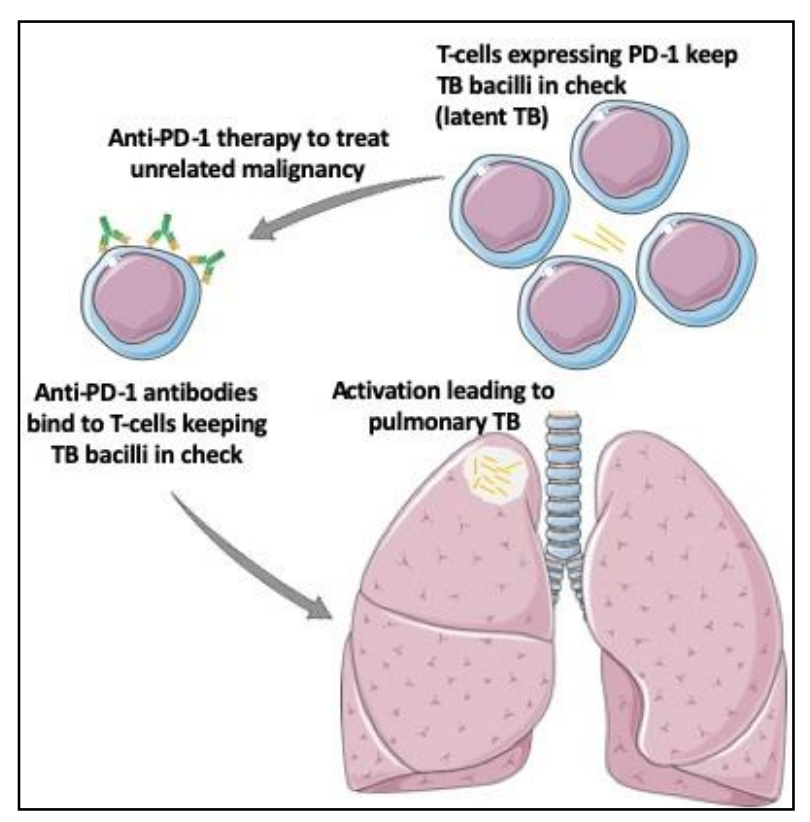

\section{Clinical caution}

In light of all these studies, it is imperative that physicians test for latent TB before starting patients on ICPIs. This caution is especially targeted to physicians practicing in countries where TB is endemic. The established way to test for asymptomatic latent TB is by Interferon- $\nu$ release assays such as the QuantiFERON-TB Gold test (10). Newer methods such as host gene expression profiles are also being studied as possible ways to diagnose latent TB $(11,12)$.

It must be stated here that even if a diagnosis of latent TB is established, the potential risk to benefit ratio should be taken into consideration on a case-by-case basis. Longer follow ups of patients and studies in pre-clinical models are likely to throw further light on the possible risk of infections in patients on ICPIs. 


\section{References}

1. Two Win Nobel for Immune Regulation Discoveries. Cancer Discov. 2018

2. Takata S, Koh G, Han Y et al. Paradoxical response in a patient with non-small cell lung cancer who received nivolumab followed by anti-Mycobacterium tuberculosis agents. J Infect Chemother. 2018

3. Infectious complications associated with the use of immune checkpoint inhibitors in oncology: reactivation of tuberculosis after anti PD-1 treatment. [editorial]. Clin Microbiol Infect 2018;24(3):216.

4. Lee JJ, Chan A, Tang T. Tuberculosis reactivation in a patient receiving anti-programmed death-1 (PD-1) inhibitor for relapsed Hodgkin's lymphoma. Acta Oncol. 2016;55:519-520.

5. Kohei Fujita, Tsuyoshi Terashima, Tadashi Mio. Anti-PD1 Antibody Treatment and the Development of Acute Pulmonary Tuberculosis, Journal of Thoracic Oncology, 2016,2238-2240,1556-0864

6. Yi-Chun Chu, Ke-Chin Fang, Hsing-Chun Chen, Yi-Chen Yeh, Chih-En Tseng, Teh-Ying Chou, Chun-Liang Lai. Pericardial Tamponade Caused by a Hypersensitivity Response to Tuberculosis Reactivation after AntiPD-1 Treatment in a Patient with Advanced Pulmonary Adenocarcinoma, Journal of Thoracic Oncology, 2017,e111-e114,1556-0864

7. van Eeden Ronwyn, Rapoport Bernardo L., Smit Teresa, Anderson Ronald. Tuberculosis Infection in a Patient Treated With Nivolumab for Non-small Cell Lung Cancer: Case Report and Literature Review Frontiers in Oncology, 2019, 659

8. Daniel L. Barber, Shunsuke Sakai, Ragini R. Kudchadkar et al. Tuberculosis following PD-1 blockade for cancer immunotherapy, Science Translational Medicine, 2018

9. Ahmed A, Adiga V, Nayak S et al. Circulating HLA-DR+CD4+ effector memory T cells resistant to CCR5 and PD-L1 mediated suppression compromise regulatory T cell function in tuberculosis. PLoS Pathog. 2018;14:e1007289.

10. Mazurek GH, Jereb J, Lobue P et al. Guidelines for using the QuantiFERON-TB Gold test for detecting Mycobacterium tuberculosis infection, United States. MMWR Recomm Rep. 2005;54:49-55.

11. Sambarey A, Devaprasad A, Mohan A et al. Unbiased Identification of Blood-based Biomarkers for Pulmonary Tuberculosis by Modeling and Mining Molecular Interaction Networks. EBioMedicine. 2017;15:112-126.

12. Rakshit S, Adiga V, Nayak S, Sahoo PN, Sharma PK, van Meijgaarden KE, Dhar C, Souza GD, Finak G, De Rosa SC, Ottenhoff TH. Circulating Mycobacterium tuberculosis DosR latency antigen-specific, polyfunctional, regulatory IL10+ Th17 CD4 T-cells differentiate latent from active tuberculosis. Scientific reports. 2017 Sep 20;7(1):11948. 